\title{
Modeling of Localized Neutral Particle Sources in 3D Edge Plasmas
}

M.V. Umanasky, T.D. Rognlien, M.E. Fenstermacher, M. Borchardt, A. Mutzke, J. Riemann, R. Schneider

This article was submitted to: $15^{\text {th }}$ International Conference on Plasma Surface Interactions in Controlled Fusion Devices, Gifu, Japan, April 27 - 31, 2002

May 23, 2002

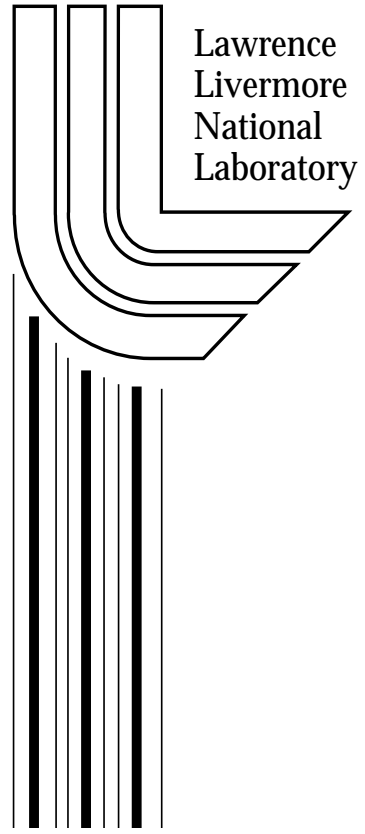




\section{DISCLAIMER}

This document was prepared as an account of work sponsored by an agency of the United States Government. Neither the United States Government nor the University of California nor any of their employees, makes any warranty, express or implied, or assumes any legal liability or responsibility for the accuracy, completeness, or usefulness of any information, apparatus, product, or process disclosed, or represents that its use would not infringe privately owned rights. Reference herein to any specific commercial product, process, or service by trade name, trademark, manufacturer, or otherwise, does not necessarily constitute or imply its endorsement, recommendation, or favoring by the United States Government or the University of California. The views and opinions of authors expressed herein do not necessarily state or reflect those of the United States Government or the University of California, and shall not be used for advertising or product endorsement purposes.

This is a preprint of a paper intended for publication in a journal or proceedings. Since changes may be made before publication, this preprint is made available with the understanding that it will not be cited or reproduced without the permission of the author. 


\title{
Modeling of Localized Neutral Particle Sources in 3D Edge Plasmas
}

\author{
M.V.Umansky,T.D.Rognlien,M.E.Fenstermacher, \\ Lawrence Livermore National Lab, \\ Livermore, CA 94550, USA
}

\author{
M.Borchardt,A.Mutzke,J.Riemann,R.Schneider, \\ Max-Planck-Institut fur Plasmaphysik, \\ D-17491 Greifswald, Germany
}

\author{
L.W.Owen, \\ Oak Ridge National Laboratory, \\ Oak Ridge, TN 37830, USA
}

May 23, 2002

\begin{abstract}
A new edge plasma code BoRiS [1] has a fully 3D fluid plasma model. We supplement BoRiS with a 3D fluid neutral model including equations for parallel momentum and collisional perpendicular diffusion. This makes BoRiS an integrated plasma-neutral model suitable for a variety of applications. We present modeling results for a localized gas source in the geometry of the NCSX stellarator. $\quad 52.35$.Py
\end{abstract}




\section{Introduction}

The $3 \mathrm{D}$ code BoRiS $[1]$ is being developed as a comprehensive numerical tool for modeling of edge plasmas in 3D geometry, in particular in stellarators. BoRiS has a fully 3D fluid plasma model, and it is becoming capable of reproducing $1 \mathrm{D}$ and 2D plasma solutions [2] from the 2D edge codes such as $\mathrm{B} 2$ [3] and UEDGE [4]. However a plasma model alone is insufficient to address many problems in the edge plasma where the neutral particles strongly influence the plasma, thus a neutral model is needed to allow for self-consistent plasma-neutral calculations. Altough the most accurate treatment of neutral transport is provided by the Monte Carlo methods, a fluid neutral model, such as the one implemented in UEDGE $[5,6]$, usually makes a reasonably good approximation for the neutrals in the edge plasmas, and it has advantages from the numerics point of view.

In the present paper we describe implementation of the fluid neutral model in BoRiS, and its application to the NCSX stellarator geometry

\section{Neutral transport model}

\subsection{Model description}

The fluid neutral description in BoRiS closely follows the models previously implemented in the tokamak edge code UEDGE $[5,6]$. The neutral fluid consists of atoms with temperature identically equal to the ion temperature, which is determined from a single ion-neutral thermal energy equation. For the neutral fluid we solve the neutral density the neutral momentum equations.

The neutral density equation is

$$
\frac{\partial}{\partial t}\left(n_{N}\right)+\nabla \cdot\left(n_{N} \vec{V}_{N}\right)=-S_{i}+S_{r}
$$

where $S_{i}, S_{r}$ stand for ionization and recombination sources.

For the neutral parallel momentum equation there are two options: the full Navier-Stokes equation and a reduced (diffusive) model.

The Navier-Stokes equation is

$$
\frac{\partial}{\partial t}\left(m n_{N} V_{\| N}\right)+\nabla \cdot\left(m n V_{N} V_{\| N}-\hat{\eta}_{N} \vec{\nabla} V_{\| N}\right)=-\nabla_{\|} p_{N}+
$$




$$
m_{i} n_{i} n_{n} K_{c x}\left(V_{\|}-V_{\| N}\right)+m_{i} S_{r} V_{\|}-m_{i} S_{i} V_{\| N}
$$

where $V_{\|}$is the ion flow velocity.

The diffusive approximation for the parallel velocity is

$$
V_{\| N}=-D_{N}\left(\nabla_{\|} n_{N} / n_{N}+\nabla_{\|} T_{i} / T_{i}\right)+V_{\|}
$$

which corresponds to neglecting all but two terms in the Navier-Stokes equation.

And the perpendicular neutral velocity is always calculated in the diffusive approximation

$$
V_{N \perp}=-D_{N}\left(\nabla_{\perp} n_{N} / n_{N}+\nabla_{\perp} T_{i} / T_{i}\right)+V_{\perp}
$$

In the diffusive model there is flux limiting of the form

$$
j_{N} \rightarrow j_{N} /\left[1+\alpha\left(\frac{j_{N}}{n_{N} V_{T i} / 4}\right)^{\gamma}\right]^{1 / \gamma}
$$

\subsection{Benchmark with UEDGE}

To verify the performance of the fluid neutral model in BoRiS, we conducted a series of $1 \mathrm{D}$ and 2D benchmarks with the 2D edge code UEDGE. Figure (1) presents results of a calculation with BoRiS and UEDGE for neutral density distribution for a sample problem in a 2D box geometry. Neutral particles are assumed injected at a rate 1000 Amperes/m through the inlet at the top boundary; at the remainder of the top boundary the normal neutral flux is set zero, on the other three sides of the box the neutral density is set at $1 \times 10^{14} \mathrm{~m}^{-3}$. Plasma parameters are assumed constant with a temperature of $10 \mathrm{eV}$, density of $1 \times 10^{20} \mathrm{~m}^{-3}$ and flow velocity of $V_{x}=5 \times 10^{4} \mathrm{~m} / \mathrm{s}$.

Both BoRiS and UEDGE were used to solve this problem with the diffusive neutral model and same models for the atomic physics rates. The resulting neutral density plumes shown in Fig. (1) are very similar, but not identical due to different meshes and finite-differencing used. 


\section{Application to NCSX geometry}

\subsection{Physical motivation}

The National Compact Stellarator Experiment (NCSX) [8] is being planned in the US as a new major fusion experiment. It is known that control of neutrals is essential for good plasma performance in stellarators [7]. The main sources of neutrals are plasma-facing components such as limiters and target plates. As it is planned to install poloidal limiters on the inner side of the "bullet-like" section [9], the neutrals will originate at those locations. If poorly confined, the recycling neutrals can penetrate deep into the main plasma and cause prohibitively large power loss by charge-exchange, radiation and convection. This is particularly an issue for NCSX where the core plasma width is rather small in the "banana-like" toroidal sections. This motivates calculation of neutral penetration into the core plasma for NCSX geometry, assuming some realistic profiles of background plasma density and temperature.

\subsection{Grid generation}

As BoRiS operates in the magnetic coordinates [1], to do calculations for NCSX geometry one needs first to calculate the magnetic coordinate representation for NCSX. A method for calculating the mapping between the real coordinates $\left(R, Z, \Phi_{g}\right)$ and the magnetic coordinates $(s, \theta, \phi)$ from given magnetic field using line tracing was developed by Boozer and coworkers $[10,11]$. We use actual magnetic field data [12] found for NCSX from magneto-hydrodynamic (MHD) free boundary calculations with numerical codes VMEC [13, 14] and MFBE [15]. We neglect the stochastic field regions found by line tracing outside of NCSX last closed magnetic surface (LCMS) [16], and consider only the inner part of NCSX flux tube where line tracing indicates existence of "good" flux surfaces [16]. The metric data from the "good" flux surfaces region are then extrapolated outside of LCMS to provide a model for NCSX geometry both inside and outside of LCMS. Using a line tracing method similar to $[10,11]$ we calculate the magnetic coordinates for NCSX from the data. Then we use several leading Fourier amplitudes of this mapping to construct a simplified model of NCSX geometry which provides a good qualitative and quantitative match to the shape and dimensions of NCSX flux tubes, as shown in Fig. 2. This model of NCSX geometry is used 
in the calculations described below.

\subsection{Code setup}

The computational domain for these calculations represents a one period of NCSX flux tube region between two flux surfaces, where the outer surface represent the outer wall, and the inner surface is close enough to the magnetic axis to allow for setting of a simple boundary condition for the neutrals there. For the background plasma we consider temperature and density profiles which are dependent on the radial flux coordinate $s$ only. The normalized radial profiles are taken in the form

$$
\begin{array}{rr}
T / T_{0}=n / n_{0}=\left[2-(3 / 2 s)^{2}\right], & 0 \leq s \leq 2 / 3 \\
T / T_{0}=n / n_{0}=\exp (2-3 s), & 2 / 3 \leq s \leq 1
\end{array}
$$

where the normalization parameters $T_{0}$ and $n_{0}$ are the LCMS values (see Fig. $3)$.

The boundary conditions for the neutrals are taken as follows: On the inner boundary of the domain $(s=0)$, a zero neutral flux condition is set since very few neutral particles are supposed to get that far into the plasma. The outer boundary $(s=1)$ also has a zero flux condition which represents a perfect wall, except for an area $0.4 \leq \theta, \phi \leq 0.6$ where a fixed neutral density

$n_{N}=1 \times 10^{18} 1 / \mathrm{m}^{3}$ is set. This region simulates a source of neutral gas localized at the inner mid-plane part of the "bullet" cross-section.

\subsection{Calculation results}

We calculated the 3D distribution of neutral density for two different plasma background temperature and density distributions: (a) $T_{0}=50 \mathrm{eV}$ and $n_{0}=1 \times 10^{19} \mathrm{~m}^{-3}$, and (b) $T_{0}=50 \mathrm{eV}$ and $n_{0}=0.5 \times 10^{19} \mathrm{~m}^{-3}$.

In Fig. 4 one can see contours of neutral density for three toroidal crosssestions for geometric toroidal angle values $60^{\circ}, 30^{\circ}$, and $0^{\circ}$. One can observe that neutral density is reduced by a factor on the order of $\sim 1000$ towards the "banana" cross-section.

In Fig. 5, corresponding to case (b) with lower density of the background plasma, one can observe that the neutrals spread broader over the plasma, and the reduction towards the "banana" cross-section is not as strong as in case (a), by a factor on the order of $\sim 100$. 


\section{Conclusions}

The BoRiS code has been supplemented with the fluid neutral model. This model has been tested against the UEDGE code in 1D and 2D setups. Applying BoRiS to neutral transport calculation in the NCSX stellarator geometry

provides full 3D solution which may have important insights for NCSX design.

\section{Acknowledgements}

We would like to thank Dr. A.Grossman (UCSD) for NCSX magnetic field data. This work is performed for USDOE by Univ. Calif. LLNL under contracts W-7405-ENG-48.

\section{References}

[1] M.Borchardt et al, J. Nucl. Mater. 290-293, (2001) 546.

[2] J.Riemann et al, Hierarchy tests of edge transport models (BoRiS, $U E D G E)$ (This conference).

[3] B.J.Braams, NET report No.68 (1987).

[4] T.D.Rognlien et al, J. Nucl. Mater. 347-351, (1992) 196.

[5] T.D.Rognlien et al, Contr. Plas. Phys. 34 (1994) 362.

[6] F.Wising et al, Contr. Plas. Phys. 36 (1996) 136.

[7] P.Grigull et al, EPS Conference, Madeira, Portugal, June 18-22, 2001.

[8] G.H.Neilson et al, Phys. Plasmas 7 (2000), 1911.

[9] P.Mioduszewski, NCSX Project Physics Meeting, 31 Jan. 2002

[10] A.H.Boozer, Phys. Fluids 25 (1982), 520.

[11] G.Kuo-Petravic et al, J. Comput. Phys. 51 (1983), 261.

[12] A.Grossman, Private communication. 
[13] S.P.Hirshman et al, Comput. Phys. Commun. 43 (1986), 143.

[14] S.P.Hirshman, D.K.Lee, Comput. Phys. Commun. 39 (1986), 161.

[15] E.Strumberger, Nucl. Fusion 37 (1997), 19.

[16] A.Koniges et al, Magnetic topology of a candidate NCSX plasma boundary configuration submitted to Nucl. Fusion (2002). 


\section{Figure captions}

Figure 1: The plume test calculation results from UEDGE and BoRiS shown. The background plasma has density $1 \times 10^{20} \mathrm{~m}^{-3}$, temperature $10 \mathrm{eV}$, and flow velocity $5 \times 10^{4} \mathrm{~m} / \mathrm{s}$. The box dimensions are $1 \times 1 \mathrm{~m}$, and the neutrals are injected through the inlet at the top boundary at a rate of 1000 Amperes $/ \mathrm{m}$. At the remainder of the top boundary the normal neutral flux is set zero, on the other three sides of the box the neutral density is set at $1 \times 10^{14} \mathrm{~m}^{-3}$.

Figure 2: Three toroidal cross-sections of the 3D grid used for NCSX calculations. For the reduced design of $\operatorname{NCSX}\left(R_{m a j}=1.4 \mathrm{~m}\right)$ the grid is uniformly scaled down by a factor of 0.82 . LCMS from MFBE calculations is shown by the red line.

Figure 3: Radial profiles of normalized density and temperature for the background plasma

Figure 4: Neutral density distribution for case (a) described in section 3.4

Figure 5: Neutral density distribution for case (b) described in section 3.4 

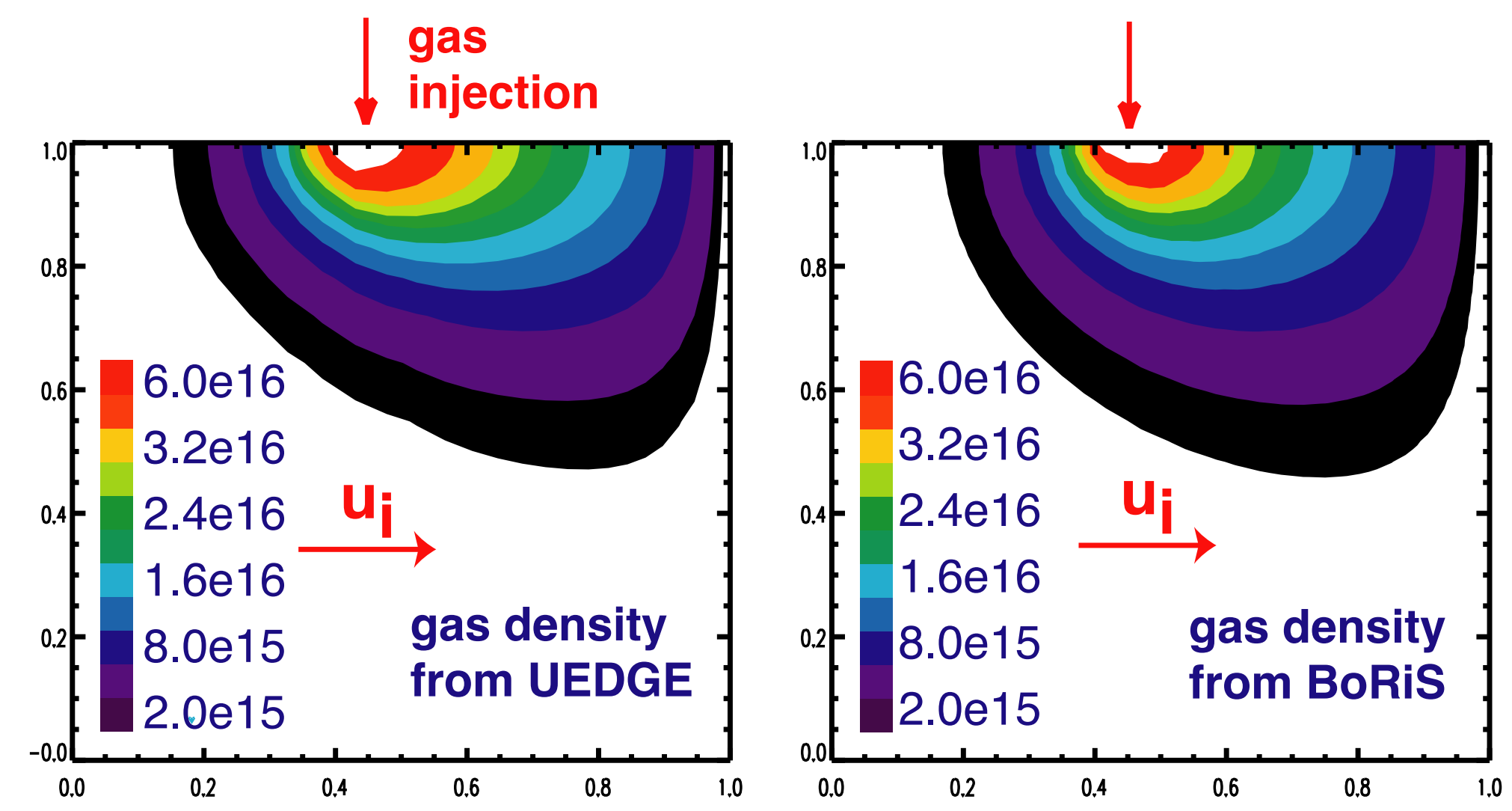

Fig. 1 


\section{Umansky}
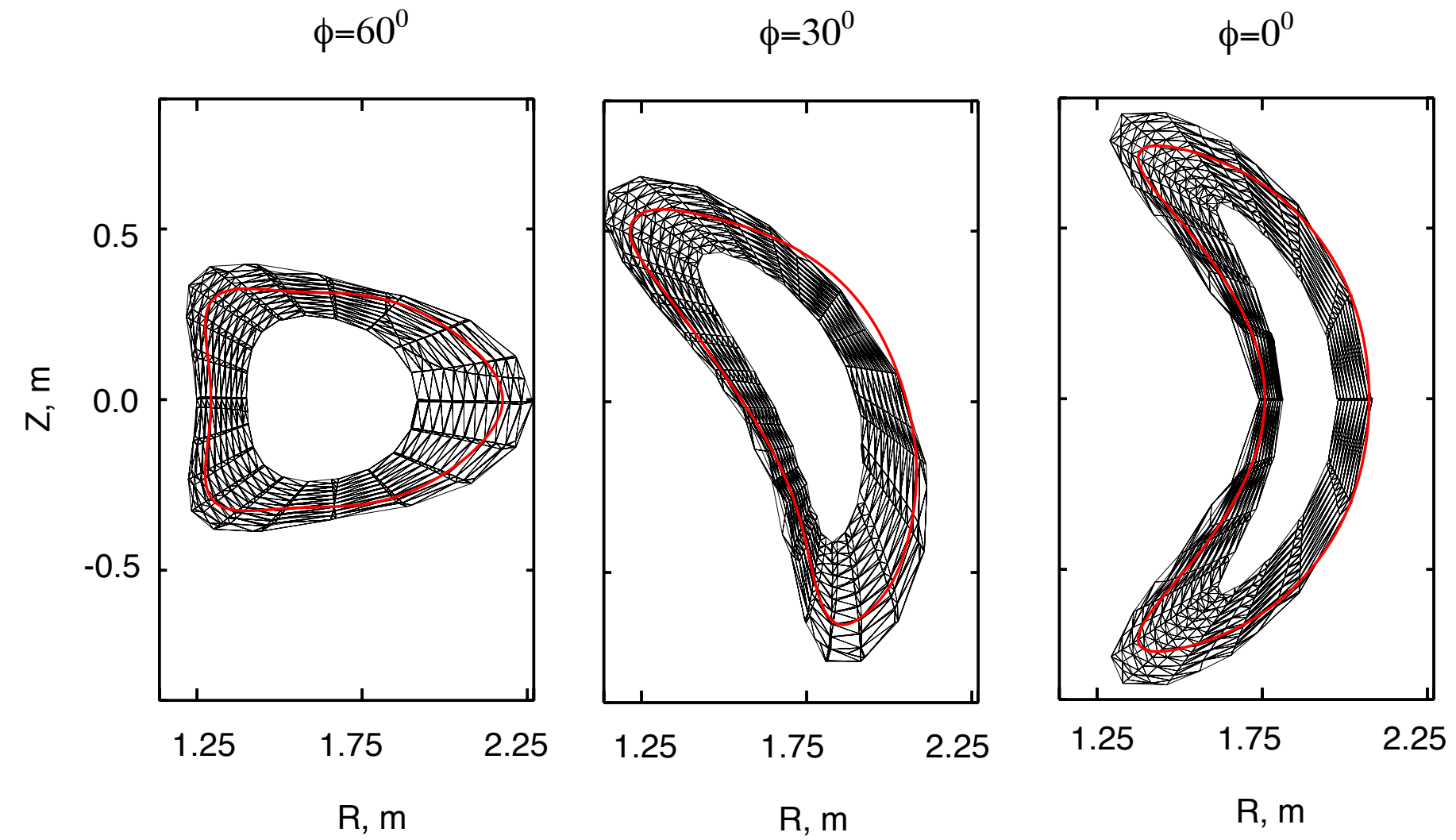

Fig. 2 
Umansky

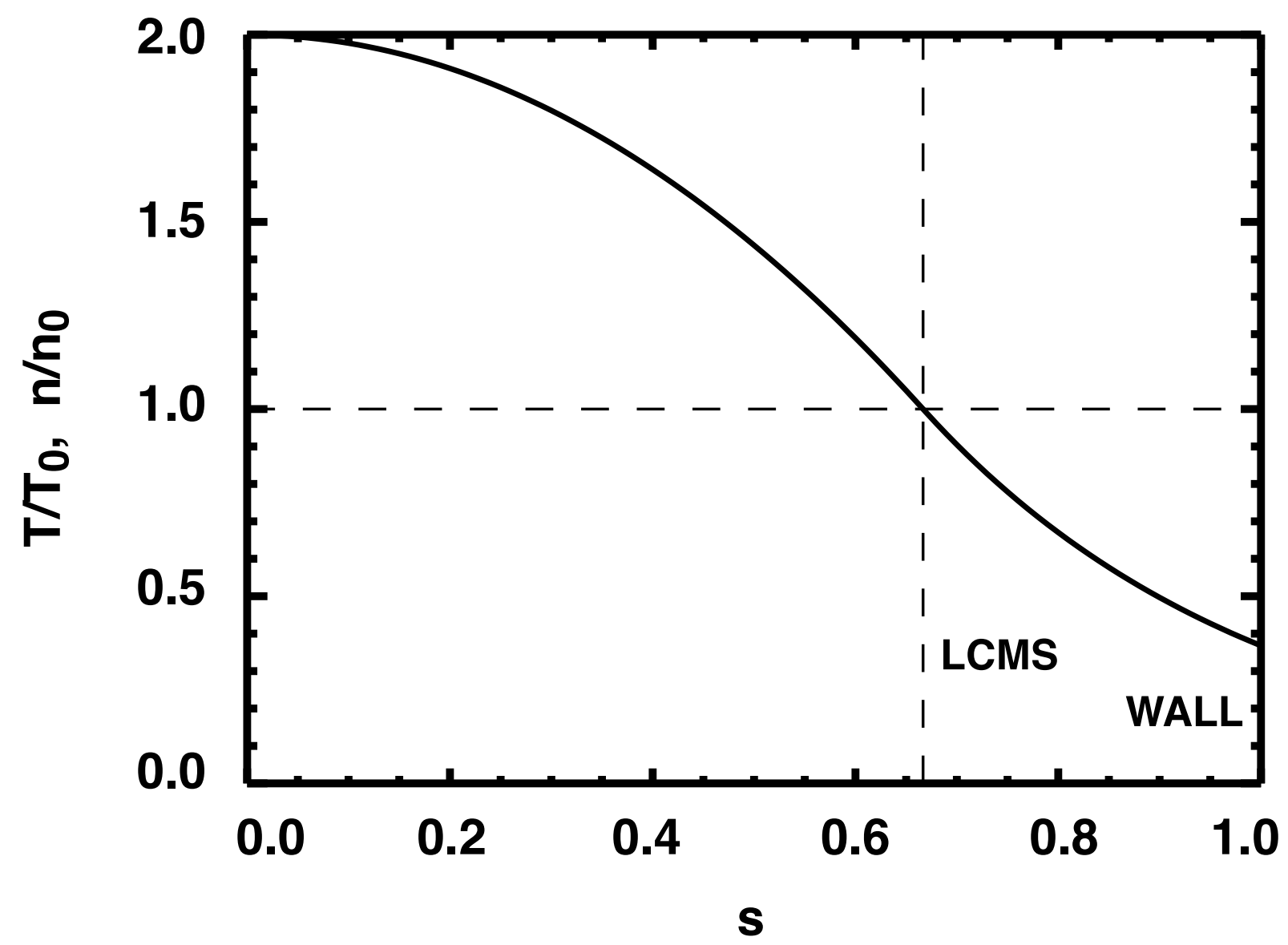

Fig. 3 
Umansky

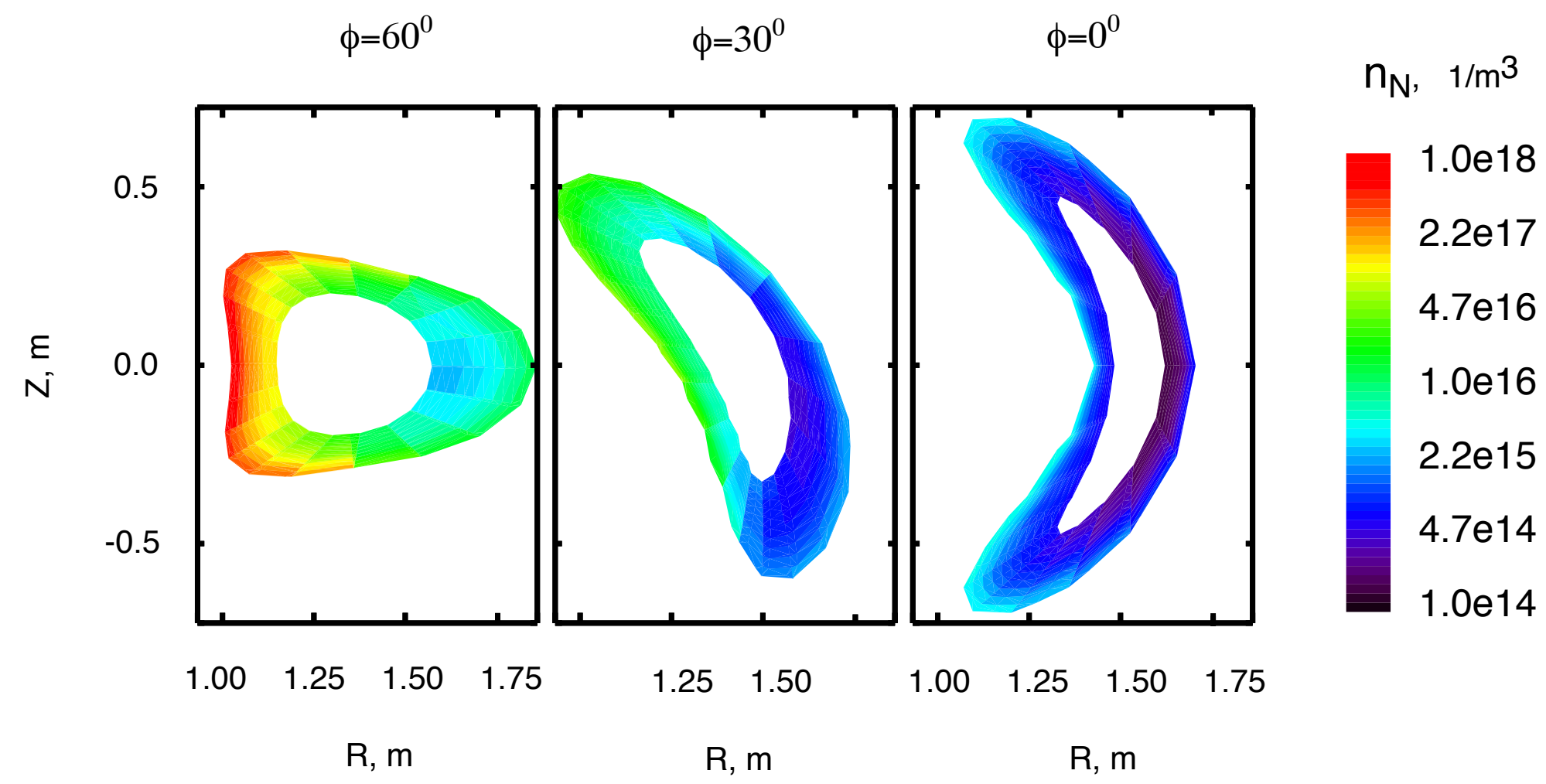

Fig. 4 


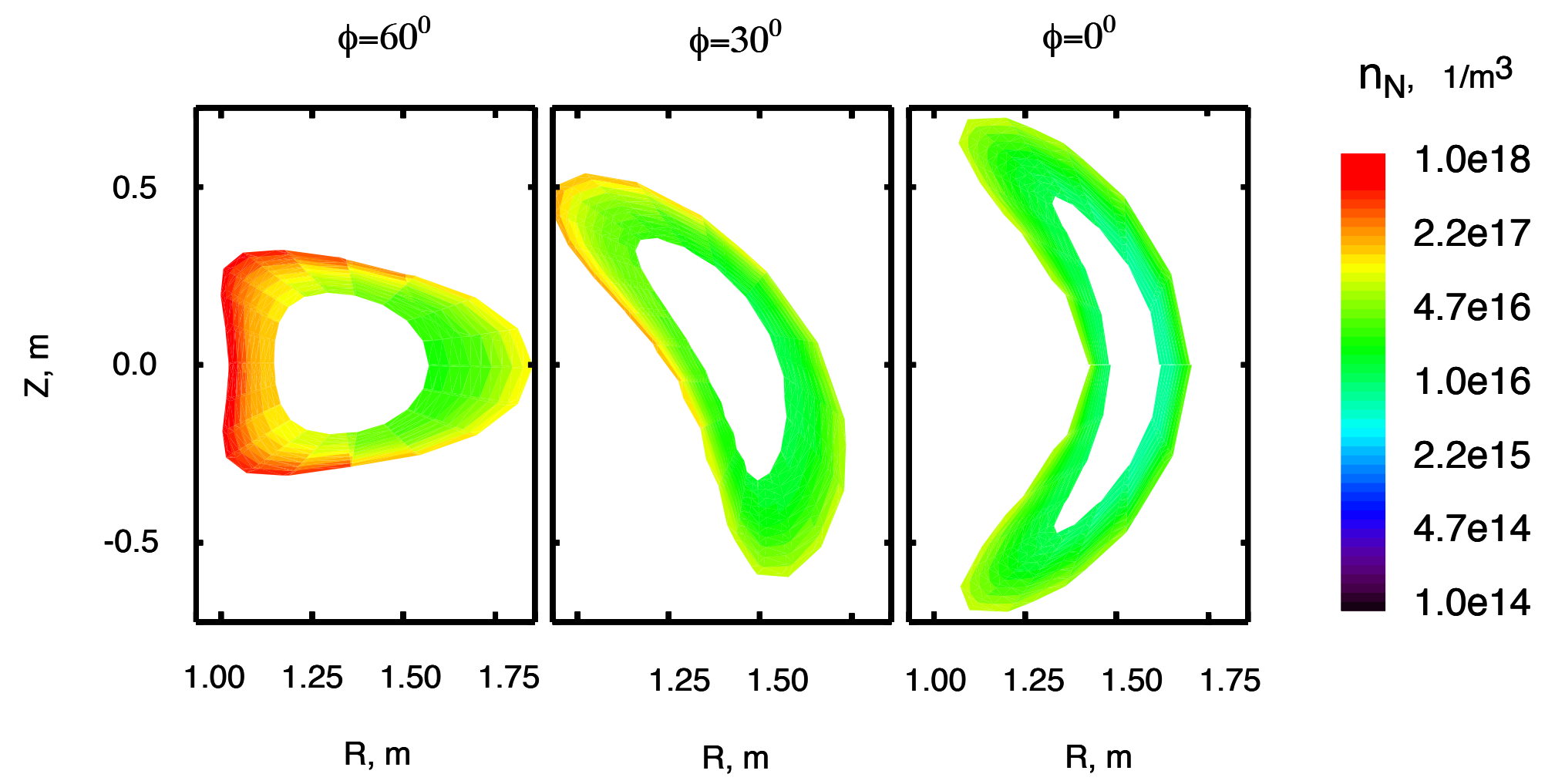

Fig. 5 